\title{
La insoportable levedad de la regulación de las profesiones del deporte The unbearable lightness of the regulation of the sports professions
}

\author{
Julián Espartero Casado \\ Universidad de León (España)
}

\begin{abstract}
Resumen: La contumaz inacción del Estado en la regulación de las profesiones del deporte, determinó la actuación legislativa de concretas Comunidades Autónomas en pro de configurar la misma en su ámbito territorial. Esto generó una problemática añadida a la ausencia de regulación estatal que incluso motivó que el legislador estatal reclamara la intervención del ejecutivo para corregir las disfunciones padecidas en este contexto. Sin embargo, ello ni ha traído la tan deseada intervención del Estado, ni tampoco ha frenado la iniciativa legislativa autonómica en este contexto, al que se han sumado cuatro nuevas leyes. Ello implica la progresiva consolidación de un modelo que, por las limitaciones competenciales autonómicas en materia de regulación profesional, no puede llevar a cabo una tarea crucial en el sistema, cual es la diferenciación en los niveles de intervención profesional a través de la necesaria coherencia entre la cualificación poseída y la actividad desempeñada. Asimismo, las normativas autonómicas existentes establecen regulaciones propias que difieren entre sí en la denominación y en los requisitos de acceso a las profesiones reguladas que ordenan, exacerban la configuración de un mercado fragmentado. Además, esta heterogeneidad regulatoria obstaculiza la vertebración de la negociación colectiva a nivel nacional en el sector, dado que formaciones exigidas por el vigente convenio colectivo para ejercer las funciones de sus categorías profesionales difieren con las que regulan estas diversas leyes autonómicas. Todo lo cual, como se justifica, solo puede solventarse con la intervención del Estado, vía del ejercicio de sus competencias exclusivas en la materia.
\end{abstract}

Palabras clave: Constitución, profesiones reguladas, unidad de mercado, negociación colectiva.

\begin{abstract}
The persistent inactivity of the State on the regulation of the sports professions, prompted the legislative action of some specific Autonomous Community for drafting this at their territorial scope, which created some added problems to this absence of the state regulation that even caused the state legislator demanded the intervention of the goverment for correcting the malfunctions endured in this context. Nevertheless, it neither has brought the much-desired intervention of the State, nor has stopped the legislative Autonomous initiative in this context, to which four new laws have been joined. This involves the progressive consolidation of a model that, because the Autonomous competency limitations with respect to professional regulation, cannot accomplish a crucial task in the system. That is the differentiation at the levels of professional intervention through the necessary coherence among the obtained qualification and the performed activity. Moreover, the existing Autonomous regulations establish some own regulations which are different each other about denomination and requisites of access to the regulated professions that order and exacerbate the configuration of a fragmented market. What is more, this regulatory heterogeneity obstructs the realization of the collective bargaining of national level in the sector, since required occupational trainings by the current collective agreement in order to practice the roles of their professions differ with those that regulate these various Autonomous laws. All this, as justified, can be overcome only with the intervention of the State, via the practice of its exclusive powers over this matter.
\end{abstract}

Key words: Constitution, regulated professions, market unity, collective bargaining.

\section{Introducción}

En mayo de 2020, el Consejo Superior de Deportes (CSD) publicó su «Anuario de Estadísticas Deportivas 2019» y, entre los principales resultados arrojados, se encuentran los relativos al empleo vinculado al deporte, significándose expresamente que «se trata del empleo que se desarrolla en empresas dedicadas a actividades deportivas, tales como la gestión de instalaciones, las actividades de los clubs y gimnasios entre otros, (...) así como el que se corresponde con las ocupaciones de (...) entrenadores o instructores deportivos». Asimismo, las cifras relativas a estos datos revelaban que el volumen medio anual de empleo vinculado al deporte «ascendió en 2019 a 219,9 mil personas, lo que supone en términos relativos un 1,1\% del empleo total en la media del periodo anual». Destacándose, igualmente, que «(...) el empleo vinculado al deporte se caracteriza también por una mayor formación académica superior a la media, presentando tasas de educación superior más elevadas que las observadas en el conjunto nacional, 53.5\% frente al 43.8\%» (CSD, 2020, p.17).

Fecha recepción: 02-08-20. Fecha de aceptación: 18-09-20 Julián Espartero Casado

jespc@unileon.es
De estos datos oficiales bien debe admitirse la confirmación de que los servicios deportivos ocupan por derecho propio un importante lugar en la actividad económica y el empleo en España (Martínez-Lemos \& Hontoria-Hernández, 2019). A su vez, dichas cifras son extraordinariamente reveladoras de que, detrás de la creciente demanda de estos servicios, se encuentra un relevante interés social de la ciudadanía española en la práctica de la actividad física y el deporte (Canales-Lacruz \& Rey-Cao, 2018; Martín, Espada, Moscoso, Jiménez, Santacruz \& Jiménez, 2018). Ello contrasta fuertemente, sin embargo, con la realidad de que la organización y regulación de los servicios profesionales responsables de la bonanza económica del sector, siguen constituyendo la asignatura pendiente del legislador estatal en materia de deporte, en cuanto que persiste su recalcitrante renuencia a abordar la regulación del ejercicio de las profesiones deportivas (Garrigós, 2002; Jiménez, 2001; Palomar, 2000; Pastor, 1997).

Esto, a su vez, ha venido a originar una prolífica reacción normativa autonómica, dirigida a la regulación de las profesiones deportivas en sus correspondientes territorios. Empero, esta notable iniciativa legislativa, por su propia naturaleza, no sólo no ha podido cubrir las necesidades que demandan la regulación del ejercicio profesional en el sector, sino que, además, ha generado una problemática añadida a 
esta ausencia de regulación estatal (Consejo General de Colegios Oficiales de Licenciados en Educación Física y en Ciencias de la Actividad Física y del Deporte -Consejo COLEF-, 2017; Espartero, 2016), que incluso motivó que el Congreso de los Diputados reclamara la intervención del ejecutivo para corregir las disfunciones padecidas en este contexto. En efecto, aprobada por unanimidad, la Proposición no de Ley (PNL) relativa a la regulación y ordenación de las profesiones del Deporte, declaraba que «El Congreso de los Diputados insta al Gobierno a que proceda a unificar y conferir un marco general e igualitario de las profesiones del deporte, respetando estrictamente las competencias autonómicas, que otorgue seguridad jurídica a los profesionales, clasificando las categorías profesionales, especificando las funciones y atribuciones propias de cada una y que determine cómo debe acreditarse dicha cualificación para acceder legalmente al ejercicio de la profesión del deporte, buscando la mejora y protección de la salud y la seguridad ciudadana» (Boletín Oficial de las Cortes Generales, 2017, p. 24).

Desafortunadamente, y como resulta conocido, esta PNL no sólo no ha alcanzado su pretendido objetivo de la publicación de una ley estatal que propicie un marco regulador «general e igualitario de las profesiones del deporte», es que tampoco ha conseguido, siquiera, atenuar la loable y desparpajada iniciativa legislativa del legislador autonómico en la materia, ante la inacción del poder público estatal. Como lo demuestra el hecho de que, tras la promulgación de la PNL, las Comunidades Autónomas (CCAA) de Murcia, Aragón, Castilla-León y Navarra, han publicado leyes que, con carácter general o específico, regulan las profesiones del deporte en su ámbito territorial. Viniendo así a sumarse a las ya existentes en los territorios de Cataluña, La Rioja, Extremadura, Andalucía y Madrid. De modo que son ya nueve, las CCAA que gozan de una regulación autonómica individualizada de concretas actividades profesionales en el ámbito de la actividad física y del deporte.

Esta situación, pues, supone un incremento de la problemática aludida y cuyos principales aspectos serán objeto de exposición en el presente trabajo.

\section{El objetivo: la necesaria protección de la salud y seguri- dad de los usuarios mediante la cualificación profesional de los servicios}

Interesa aquí destacar que cuando la PNL alumbrada por el Congreso de los Diputados insta al Estado a regular el marco de los servicios profesionales de la actividad física y del deporte, lo hace determinando claramente la forma y la finalidad de la misma. Así, esta regulación debe llevarse a cabo dotando al sistema de unidad y de un carácter general e igualitario para otorgar seguridad jurídica a los profesionales del sector, clasificando sus categorías profesionales, especificando sus atribuciones y la forma de acreditar su cualificación profesional. Todo lo cual debe de tener como causa final, la búsqueda del interés público que se concita en la salud y seguridad de los usuarios (Palomar, 2019). Lo que supone una nítida alineación con los postulados que marcara la decisiva Sentencia del Tribunal Constitucional (en adelante STC) 194/1998, al indicar que aquel interés público perseguido tiene como medio necesario el control de acceso a la prestación de los servicios profesionales en la actividad físico-deportiva, sobre la base de la tenencia de una adecuada cualificación, en cuanto que ello se proyecta como una auténtica y decisiva garantía para la seguridad y la salud de los practicantes de la actividad deportiva (Jiménez, 2001; Landaberea, 2008; Palomar 2000).

Es más, es esta finalidad tuitiva la que de forma natural y necesaria comporta la pertinente exigencia de una calidad en los servicios profesionales que se desenvuelven en concretos planos del contexto deportivo. Calidad que debe hallar su verificación en el desarrollo de una praxis profesional cualificada tal, que efectivamente permita el confiado sustento de ese objetivo garantista, de ese deber cuidado que demanda la seguridad y la salud de los usuarios. Lo que exige que estos servicios sólo puedan y deban ser prestados por personal que posea la debida cualificación y con ello garantizar que aquellos derechos no se vean afectados por el ejercicio de sus actividades profesionales (Garrigós, 2001; González \& Esteban, 2015; Lolli, 1997; Madrera, Sánchez, 2012).

Debe, por tanto, implementarse un marco regulatorio coherente con tales postulados, teniendo en cuenta, además, que la Constitución no sólo contempla una norma general de garantía del derecho a la salud -artículo 43-, sino que también invoca la protección del consumidor: «1. Los poderes públicos garantizarán la defensa de los consumidores y usuarios, protegiendo, mediante procedimientos eficaces, la seguridad, la salud y los legítimos intereses económicos de los mismos» (art. 51). Se trata, pues, de una protección que se despliega frente a los riesgos que pueden afectar a la propia salud y seguridad, provocados, no ya por la posible incidencia de patología o enfermedad, sino por otras actividades humanas (Cimmino, 2017). En su consecuencia, el objetivo de seguridad, vinculado a la protección de la salud, tiene un respaldo expreso en la normativa de consumidores y usuarios aplicable en tanto en cuanto la mayor parte de la práctica deportiva se realiza en instalaciones o con servicios de personas especializadas que son los llamados a orientar la misma y a hacer compatible las características personales con el esfuerzo y el nivel que corresponde realizar a los poderes públicos (Landaberea, 2008; Lolli, 1997; Palomar, 2019).

En este sentido, la Comisión Europea señalaba -en su comunicación «Desarrollo de la dimensión europea en el deporte», de 18 de enero de 2011-, entre otros asuntos prioritarios, que «Los Estados miembros y el movimiento deportivo reconocen la necesidad de disponer de personal más cualificado en el sector del deporte. (...) Es necesario mejorar (...) la claridad de las cualificaciones necesarias para ejercer las profesiones reguladas relacionadas con el deporte» (aptdo. 2.2). Lo que ha de ponerse necesariamente en conexión con la Directiva 2018/958/UE del Parlamento Europeo y del Consejo, de 28 de junio de 2018, relativa al test de proporcionalidad antes de adoptar nuevas regulaciones de profesiones (Terol, 2019). La finalidad de esta norma radica en establecer normas aplicables a las evaluaciones de proporcionalidad que los Estados miembros deben realizar antes de introducir nuevas regulaciones profesionales o de modificar las existentes, para garantizar el buen funcionamiento del mercado interior, a la vez que se garantiza la transparencia y un nivel elevado de protección de los consumi- 
dores. Así pues, se impone a los Estados miembros la obligación de asegurarse de que las disposiciones legales, reglamentarias o administrativas que restrinjan el acceso a las profesiones reguladas o su ejercicio, sean ya existentes o que se pretendan introducir, estén justificadas por objetivos de interés público: «(...) orden público, seguridad y salud públicas, o por razones imperiosas de interés general (...)» (aptdo. 17). Asimismo, dicha obligación se extiende a velar que las restricciones a que puedan dar lugar estas disposiciones sean adoptadas por los Estados miembros tomando en consideración su repercusión, entre otras razones, «en la calidad del servicio prestado» (art. 7.2 b).

Es evidente, pues, que la primera y principal manifestación de la calidad de los servicios en la actividad física y el deporte radicará en que su ejercicio profesional resulte estar presidido por la coherente conexión entre profesión y titulación a través de la efectiva correspondencia entre el ámbito de intervención con la adecuada cualificación (Bujanda, 2017; Consejo COLEF, 2018; Feu, García-Rubio, Antúnez \& Ibáñez, 2018; Miñana-Signes \& Monfort-Pañego, 2020; Sans \& Inglés, 2020). Consideración esta que se ve enfáticamente patentizada, particular y muy ejemplarmente, en el caso de poblaciones con problemas de salud, diversidad funcional o con capacidades diferentes (Gómez, 2019; González \& Contreras, 2003). De tal manera que las necesidades de estos colectivos en materia de ejercicio físico como apoyo a las terapias o tratamientos implementados por profesionales sanitarios, requieren competencias y atribuciones profesionales que cualifiquen a sus titulares para dirigir el ejercicio físico tanto con personas sanas como con personas con patologías, es decir, para intervenir con personas con enfermedades o lesiones mediante ejercicios físicos adecuados a sus características y necesidades, y por supuesto, exclusivamente mediante la aplicación de las ciencias de la actividad física y del deporte (Consejo COLEF, 2019a).

Precisamente, dichas consideraciones constituyeron el objeto de la consulta que elevó el Consejo COLEF, el 14 de Octubre de 2019, a la Subdirección General de Ordenación Profesional del Ministerio de Sanidad, Consumo y Bienestar Social, con el objetivo de aclarar definitivamente cualquier duda relativa a las competencias profesionales del el Educador Físico en dicho contexto. Dicha Subdirección General de Ordenación Profesional evacuó informe, el 28 de octubre, en el que se declaraba expresamente que «Los Educadores/as Físico Deportivos/as pueden dirigir, supervisar y evaluar la práctica de ejercicio físico, adaptado a las características, necesidades y preferencias de estas personas, contribuyendo a la adherencia a las prescripciones de los profesionales sanitarios y al mantenimiento de la práctica de la actividad física regular».

La contundencia clarificadora de este informe pone en evidencia, sin embargo, las distorsiones que afectan a la realidad del modelo que implementa el conjunto de regulaciones autonómicas sobre esta materia y su consiguiente levedad. En tanto en cuanto todas determinan que las distintas actividades profesionales que regulan puedan ser realizadas, incluso en el particular contexto que se acaba de exponer de poblaciones especiales, en régimen de competencia concurrencial por profesionales que estén en posesión de titulaciones de distinto nivel académico, sino también por aquellos que posean una cualificación derivada de la acreditación de competencias obtenidas por experiencia o aprendizajes no formales. Esta situación, que ya viene manifestándose secularmente como un problema crónico y nuclear del sector (Espartero y Palomar, 2011; Feu et al., 2018; Garrigós, 2001; Palomar, 2000; Sánchez \& Rebollo, 2000), revela la incapacidad del modelo para solventar la problemática derivada de la falta de conexión entre profesión y titulación a través de la efectiva correspondencia del ámbito de intervención con la adecuada cualificación. Lo cual sólo puede hallar su cabal y lícita corrección a través del establecimiento de las correspondientes reservas exclusivas de actividad profesional sometidas a la tenencia de una determinada titulación (Espartero, 2020; Gambau, 2019; Muñoz, 2018; Palomar, 2019; Zaragozá-Sevilla \& Martínez-Baena, 2018). Actuación esta que aparece imbricada en la competencia exclusiva del Estado y, por tanto, vedada a la actuación normativa autonómica, como se pondrá de manifiesto a continuación.

\section{Las limitadas competencias de las CCAA en materia de regulación del ejercicio profesional}

En el tratamiento de la delimitación de competencias de Estado y CCAA en la regulación del ejercicio profesional, resulta ser crucial la doctrina establecida por la jurisprudencia del Tribunal Constitucional, en cuanto que, ante la ausencia de regulación legal, ha sido la misma la encargada de desbrozar este campo configurando la distinción entre profesiones libres, profesiones reguladas y profesión titulada.

Así, la categoría de profesión titulada se definió por la STC 42/1986 como la «profesión para cuyo ejercicio se requieren títulos, entendiendo por tales la posesión de estudios superiores y la ratificación de dichos estudios mediante la consecución del oportuno certificado o licencia» (FJ 1º). Sustentándose dicha categorización jurisprudencial en que la competencia exclusiva del Estado para regular las condiciones de obtención, expedición y homologación de los títulos académicos y profesionales, se vincula directamente a la existencia misma de las profesiones tituladas y con ello, también, la determinación de las condiciones del ejercicio profesional (Fernández, 1996). De modo que «(...) corresponde al legislador, atendiendo a las exigencias del interés público y a los datos producidos por la vida social, determinar cuándo una profesión debe pasar a ser profesión titulada, y no es dudoso que, con arreglo al texto del art. 149.1.30 de la Constitución, es el legislador estatal quien ostenta esta competencia exclusiva» (STC 122/1989 FJ 3º).

Esto es así, debe insistirse en ello, con independencia de que la mayoría de los Estatutos de Autonomía hayan declarado la competencia exclusiva de sus respectivas Comunidades en materia de colegios profesionales y regulación del ejercicio profesional. Pues, dicha circunstancia debe contemplarse desde la perspectiva que marcara la STC 31/2010, afirmando que la interpretación que debe hacerse de la competencia autonómica exclusiva contenida en los diferentes Estatutos de autonomía -en relación con la regulación del ejercicio de las profesiones tituladas-, ha de ser acorde con la que ha realizado el Tribunal Constitucional en sus pronunciamientos sobre la materia. Dicha postura, que se ha mantenido inalterada desde sus inicios y a lo largo de numerosos 
pronunciamientos -SSTC 42/1981, 83/1984, 42/1986, 82/1986, 122/1989, 82/1993,111/1993,330/1994,109/2003, 154/2005,212/ 2005-, tuvo una decisiva reafirmación en la STC 201/2013, al reiterarse expresamente que en materia de ejercicio de las profesiones tituladas, «(...) la competencia autonómica se contrae al «ejercicio» de las mismas y está, además, estatutariamente subordinada a las normas generales sobre titulaciones académicas y profesionales, que se reservan al legislador estatal en los arts. 36 y 149.1.30 ${ }^{\text {a }} \mathrm{CE}$. (...), por lo que corresponde al Estado determinar, con alcance general, el concepto de profesión titulada» (FJ. 4).

Es un hecho conocido que la limitación que esto implica para la competencia de las CCAA para llevar a cabo la regulación del ejercicio profesional se vio, en principio, desbordada por diversas normas autonómicas publicadas al objeto de regular las profesiones del deporte en los territorios de sus respectivas Comunidades y trajo consigo la intervención del Estado por una posible usurpación de sus competencias. No obstante, estos conatos conflictuales fueron superados en el trámite previo a la interposición del recurso de inconstitucionalidad, a través de los acuerdos alcanzados entre las preceptivas Comisiones Bilaterales de Cooperación que hubieron de celebrarse entre Administración General del Estado y las CCAA de Cataluña (BOE $\left.n^{\circ} 40,2009\right)$ Extremadura (BOE $n^{\circ}$ 47, 2009), La Rioja (BOE $\left.n^{\circ} 55,2016\right)$, Andalucía (BOE $\left.n^{\circ} 136,2017\right)$ y Aragón (BOE n 219, 2019). En todos estos casos, los citados acuerdos alcanzados sortearon la posible inconstitucionalidad de estas normativas autonómicas, sobre la base del «entendimiento consensuado» de que las previsiones de los artículos y disposiciones contenidas en dichas Leyes que aludieran a títulos académicos no habrían de ser entendidas en el sentido de exigir tales como requisito habilitante para el ejercicio de la profesión que regulaban, sino que las mismas «(...) se refieren a los títulos aludidos en dichos preceptos en cuanto expresan la preparación en competencias y capacidades adecuadas para el ejercicio de las profesiones a las que aluden dichos preceptos» (Pascual, 2016, p.18).

Asimismo, y al efecto de eludir la interposición de recurso de inconstitucionalidad frente a estas regulaciones, todas estas Comisiones dichas coincidieron en interpretar que la exigencia de los grados de formación en competencias y capacidades para ejercer una profesión debe explicitarse en las competencias y conocimientos acreditados con las titulaciones adecuadas. Por lo tanto, esos mismos grados de formación en competencias y capacidades pueden acreditarse tanto mediante los títulos a los que en cada caso alude la Ley de que se trate $y$, de igual forma, mediante las otras titulaciones, acreditaciones o certificados de profesionalidad que resulten de las leyes estatales y del resto del ordenamiento jurídico vigente en cada momento (Correa, 2019, García, 2020, Pascual, 2016). Lo cual determina, a juicio de Muñoz (2018), que estas iniciativas legislativas autonómicas sean «normas de escaso recorrido pues su redacción parece caminar por una cuerda floja para no caer (...) en un desafío al orden constitucional de distribución de competencias entre el Estado y las CCAA» (p.130).

En efecto, la primera consecuencia de estas consideraciones que se acaban de exponer, es que estas regulaciones autonómicas, a pesar de la denominación de las mismas, no pueden ser identificadas como regulaciones del ejercicio profesional en el sentido propio o estricto configurado por el Tribunal Constitucional (Espartero, 2020). De forma que como afirmara la STC 122/1989, «(...) resulta obligado señalar (...) que la sujeción a determinadas condiciones o el cumplimiento de ciertos requisitos para poder ejercer una determinada actividad laboral o profesional es cosa bien distinta y alejada de la creación de una profesión titulada (...). Es así posible que, dentro del respeto debido al derecho al trabajo y a la libre elección de profesión u oficio (art. 35 de la Constitución), y como medio necesario para la protección de intereses generales, los poderes públicos intervengan el ejercicio de ciertas actividades profesionales (...)» (FJ. 3).

Por tanto, es claro que en la regulación autonómica relativa a las profesiones del deporte refiere a profesiones reguladas, en cuanto que dichas normativas implican la sujeción de las mismas a determinadas condiciones o a la exigencia del cumplimiento de ciertos requisitos, de modo que lo que establecen son medidas regulatorias que pretendan garantizar la posesión de una «capacitación oficial» (Pascual, 2016) -no un «título académico profesional», que es precisamente lo que permite identificar a las profesiones tituladas- por parte de quienes pretendan acceder al ejercicio de las actividades que en las mismas se determinan (Calvo, 2019). Todo lo cual, como se viene significando, no puede identificarse con lo que es regular el ejercicio de una profesión, pues, como ilustrativamente señalara la STC 386/1993, «Regular la actividad y sus efectos es (...), cuando concurre un interés público (...), una atribución del legislador. Pero regular una actividad no es, forzosamente, regular una profesión (...). La profesión incluye, generalmente, la realización de un disperso haz de actividades cada una de las cuales es susceptible, en su caso, de tener una diferente regulación (...); la regulación de una de esas actividades no es, por consiguiente, la de la profesión en su conjunto (...). Es perfectamente posible que por una sola profesión se realice una diversidad de actividades sometidas a un diferente régimen regulador, sin que por ello deban confundirse la regulación de la actividad con la de la profesión» (FJ. $3^{\circ}$ ).

En su consecuencia, las regulaciones autonómicas en la ordenación de las profesiones del deporte, a pesar del loable afán perseguido, no pueden ir más allá, so pena de sobrepasar el ejercicio de sus competencias estatutarias (Correa, 2019; Gambau, 2019; Muñoz, 2018), de establecer que el acceso al ejercicio de estas actividades profesionales o laborales reguladas se condicione a la posesión de titulaciones de diferente nivel académico, certificados de profesionalidad, diplomas federativos, así como otras titulaciones, acreditaciones o certificados de profesionalidad que resulten de las leyes estatales y del resto del ordenamiento jurídico vigente en cada momento. Toda vez que el sometimiento del ejercicio de la profesión a la tenencia de un título académico de educación superior, con la correspondiente atribución de la exclusividad de la reserva de actividad profesional de que se trate, sólo puede serlo mediante el establecimiento de profesión titulada y esto, como se ha reiterado, es una competencia exclusiva del Estado (Correa 2019, Palomar, 2019).

De ahí que, en rigor, el conjunto de regulaciones autonómicas vigentes no regulan el ejercicio profesional en el deporte, sino que ateniéndose a su acervo competencial iden- 
tifican «actividades que requieren de cualificación para su prestación como servicio y les da denominación de profesión» (Gambau, 2019, p.19). Lo que determina su levedad, en cuanto que dichas normativas no pueden, por tanto, resolver la cuestión esencial que reside en la necesidad de conexión entre la actividad laboral o profesional desarrollada y la adecuada cualificación para llevarla a cabo. Por ello determinan que el desempeño de las actividades que regulan, lo sea en régimen de competencia concurrencial, al hacerse depender de la posesión de unas competencias que pueden acreditarse tanto a través de titulaciones académicas de distinto grado o nivel, como de acreditaciones o certificados de profesionalidad. Con lo cual se reincide en una actuación centrada en controlar el acceso al ejercicio profesional o laboral de estas actividades, sobre la base de exigir una capacitación o cualificación heteróclita, pero que no atiende a corregir la consecuencia de que, superada dicha barrera, se produzca una competencia en régimen de igualdad entre profesionales que tienen cualificaciones de distinto grado o nivel e incluso distinta naturaleza (Espartero, 2016). Lo que incentiva la desestructuración que propicia la paradójica situación que supone el establecimiento de un sistema de regulación de concretas actividades profesionales o laborales, determinando para su desempeño que el mismo nivel de intervención profesional pueda ser desenvuelto por profesionales con diferentes y desiguales niveles de formación (Estrada-Marcén, Sanz, Casterad, Simón, \& Roso, 2019; Javaloyes, 2019; Muñoz, 2018; Zaragozá-Sevilla \& Martínez-Baena, 2018).

\section{Laproblemática añadida}

Pero la problemática que depara la actual situación, no sólo se limita a las consideraciones expuestas. A ello debe añadirse, la circunstancia que señalara el Consejo COLEF (2018) de que «las profesiones que se están regulando se identifican de forma heterogénea en cada Comunidad Autónoma, de manera que las profesiones tienen diferente denominación, además de requisitos de acceso que no coinciden» (p.1). Ello supone insistir en la progresiva consolidación de un modelo que, entre otras circunstancias, genera distorsión en este ámbito, tributaria de una «desigual identificación del espacio profesional y de los dispares criterios de acceso entre las regulaciones existentes» (Gambau, 2019, p.19), provocando situaciones en las que muchos profesionales y operarios del sector no pueden ejercer la prestación de sus servicios de modo uniforme en cada uno de estos territorios autonómicos, dada la citada disparidad normativa existente entre los mismos. Lo cual no deja de constituir una barrera a la libre prestación del ejercicio de su actividad, con la consecuente limitación de su movilidad (Consejo COLEF, 2018).

En efecto, la vigente situación, basada en el reconocimiento de la diversidad normativa en el marco de la unidad de mercado, puede presentar dificultades importantes derivadas de la inexistencia de referencias únicas (García, 2020). Así, actualmente, la exigencia de cualificación para acceder a la prestación de servicios profesionales en el deporte se desenvuelve en un heterogéneo contexto, conformado por CCAA en las que no existe regulación al respecto, junto con otras en las que mayoritariamente sí, pero con la particularidad reiterada de hacerlo de forma distinta entre ellas. Lo cual propicia, como se acaba de decir anteriormente, que un mismo título académico puede tener una diferente funcionalidad o competencia profesional conforme a la respectiva regulación autonómica con la que nos encontremos, determinando la realidad de un mercado excesivamente fragmentado (Correa, 2019; Espartero, 2016; Javaloyes, 2019).

En este sentido, debe recordarse que el Tribunal Constitucional ha calificado reiteradamente a las competencias exclusivas del Estado como la garantía de la unidad de mercado (Fernández, 2015). Ha de insistirse, por tanto, que esta disfunción bien puede ser corregida por la intervención del Estado, ejercitando la competencia exclusiva que le atribuye la Constitución de «La regulación de las condiciones básicas que garanticen la igualdad de los ciudadanos en el ejercicio de sus derechos y en el cumplimiento de sus deberes constitucionales» (art. 149.1.1). De modo que en esta materia de regulación del ejercicio profesional se proyecte como el adecuado elemento integrador que pueda subsanar esta fragmentación del mercado mediante el establecimiento de una regulación profesional básica, que actúe como un mínimo común denominador que armonice el ejercicio profesional de los operadores en todo el territorio nacional y soslayando los obstáculos que puedan generar la diversidad regulatoria de las CCAA(Consejo COLEF, 2018; Correa, 2019; García, 2020).

Asimismo, en tanto en cuanto esta intervención estatal no se produzca, dicha fragmentación del mercado puede propiciar la creación de marcos autonómicos de relaciones laborales, obstaculizando así la uniforme vertebración y articulación de la negociación colectiva a nivel nacional en el sector (Espartero, 2016). Singular ilustración de esta prevención es la que representa la realidad de que la diferencia de los niveles de cualificación exigibles en las diversas regulaciones autonómicas de las actividades profesionales del deporte, en muchos casos difieren con la formación o especialización exigida para ejercer las actividades que integran los grupos profesionales previstos en el vigente IV Convenio colectivo estatal de instalaciones deportivas y gimnasios, de 29 de mayo de 2018.

En tal sentido, debe tenerse en cuenta que el Real Decreto Legislativo 2/2015, de 23 de octubre, por el que se aprueba el texto refundido de la Ley del Estatuto de los Trabajadores determina que «1. Mediante la negociación colectiva o, en su defecto, acuerdo entre la empresa y los representantes de los trabajadores, se establecerá el sistema de clasificación profesional de los trabajadores por medio de grupos profesionales» (art. 21). Conforme a dicha prescripción, el citado Convenio establece cinco grupos funcionales que responden a las competencias y tareas básicas que desempeñan los trabajadores, así como, la formación o especialización exigida para ejercerlas (art. 40) y, con base en esta determinación, se regula la posibilidad del desempeño de trabajo en distintos grupos profesionales (art. 41), pudiéndose encomendar al trabajador funciones propias de un grupo profesional distinto al suyo, siempre que existan razones técnicas u organizativas que lo justifiquen y por el tiempo imprescindible para su atención (Cruz, \& Gómez, 2014).

Así las cosas, el problema que aquí se plantea es que las 
formaciones requeridas para los distintos grupos profesionales establecidos en el Convenio difieren de los requerimientos legales que realizan las distintas normativas autonómicas sobre la materia. Esta situación ya fue tempranamente avisada por el Consejo COLEF (2017) y, con la pretensión de subvenir a la misma y dentro del marco de la negociación del futuro V Convenio, ha trasladado a patronal y sindicatos una propuesta para adaptar los grupos ocupacionales del convenio del sector a la realidad de las leyes autonómicas de regulación del ejercicio profesional del deporte (Consejo COLEF, 2019b).

Sin embargo, en tanto esto no suceda, la situación puede propiciar circunstancias claramente negativas. Así, y conforme al principio de jerarquía, es clara la prevalencia de las normas legales, estatales y autonómicas, sobre lo dispuesto en convenio colectivo (Casas, 2003). En este sentido, el Tribunal Constitucional ha defendido la superioridad de la ley, incluso la posterior al convenio colectivo, al declarar que lo acordado con fuerza vinculante en la negociación colectiva puede ser modificado por ley posterior y ello porque «la Ley ocupa en la jerarquía normativa una superior posición a la del convenio colectivo, razón por la cual éste debe respetar y someterse a lo dispuesto con carácter necesario por aquélla, así como, más genéricamente, a lo establecido en las normas de mayor rango jerárquico y no al contrario» (STC 210/1990, FJ. 2). Todo lo cual lleva a colegir que ello convertiría en inaplicable el vigente Convenio nacional en las CCAA que poseen regulación legal profesional, al diferir las formaciones establecidas por el mismo para ejercer las funciones de sus grupos profesionales, en el ámbito de los señalados artículos 40 y 41, de lo que determinan estas leyes autonómicas.

Consideración esta que se ve reforzada, además, por la reiterada interpretación de la jurisprudencia del Tribunal Supremo que ha venido determinado que «Cuando el ejercicio de las funciones de una determinada actividad profesional se encuentra regulado por normas legales de carácter imperativo que para su desempeño exigen una determinada titulación académica, no es posible realizar válidamente, aun temporalmente, las tareas correspondientes sin estar en posesión de la debida titulación, en cuanto la norma imperativa prohíbe el ejercicio profesional si se carece de la misma (...)» (STS 3127/2018, FD.4).

Asimismo, habida cuenta de la diversidad de la normativa autonómica en la identificación de las actividades profesionales que se regulan, así como en los requisitos de acceso a las mismas, creemos que es inviable que se pueda llevar a cabo una adaptación de la regulación colectiva a la prolija y heteróclita regulación que contienen las vigentes regulaciones autonómicas. De momento y como es conocido hasta la fecha, son nueve. ¿A cuál de ellas habría de adaptarse la negociación colectiva? Es más, en el caso que se consiguiera llevar a cabo esta adaptación, esto pudiera suponer un nuevo menoscabo de la doctrina constitucional relativa al principio de unidad de mercado en el que el reconocimiento de la diversidad normativa entre CCAA quedara manifiestamente en entredicho, por ejemplo, cuando una normativa autonómica menos rígida o exigente en la regulación de actividades profesionales en el deporte, que puede tener su razón de ser en las peculiaridades propias del territorio en el que en principio ha de regir, pudiera extrapolarse a otro ámbito territorial en el que, precisamente, las especificidades que le son propias pueden ser la causa de la existencia de un régimen jurídico más riguroso (Tornos, 2014).

De aquí que, de nuevo, sea justificado reclamar la intervención del Estado para la solución de esta problemática. Pues sólo a través de la actuación estatal será posible una imbricación normativa básica y uniformadora en materia de regulación del ejercicio profesional y con ello el necesario sometimiento de la regulación colectiva a dicha ordenación armonizadora en materia del establecimiento de las adecuadas formaciones para acceder a las correspondientes categorías profesionales (Espartero, 2016; Gambau, 2019; Palomar, 2019).

\section{Conclusiones}

La Constitución Española, en su artículo 149.1.30, reserva al Estado la competencia exclusiva sobre regulación de las condiciones de obtención, expedición y homologación de títulos académicos y profesionales. Parece sensato, pues, que sobre la base de esta competencia, deba ser el legislador estatal el que determine cuando el ejercicio de una profesión deba someterse a la tenencia de dicho títulos, porque así lo demanda la concurrencia de un interés público de carácter constitucional, cual es la salud y la seguridad de los ciudadanos en la práctica de la actividad física y del deporte.

Es indudable que la inacción del Estado en este sentido, es la responsable de la actuación legislativa de las CCAA en pro de la consecución de esta garantía de protección y cuidado. Sin embargo, este notable empeño no puede ir más allá de los límites que marcan las competencias estatutarias de las mismas, so pena de incurrir en inconstitucionalidad. Esta insuficiencia competencial de las CCAA, pues, impide la completa satisfacción de este reiterado interés público, pues su esfuerzo normativo no puede reparar la falla endémica del sistema que depara la desconexión entre titulación y reserva profesional propia. Y si no puede implementarse una diferenciación en los niveles de intervención profesional que propicie la necesaria coherencia entre la cualificación poseída y la actividad desempeñada, entonces, la provisión regulada de los servicios profesionales en el deporte continuará viéndose afectada de desestructuración y de falta de la necesaria calidad para el cumplimiento de su finalidad tuitiva (Espartero, 2020; Madrera et al., 2015; Muñoz, 2018; Palomar, 2019).

Asimismo, y como se ha dicho, si se mantiene el panorama normativo vigente, de manera que tengamos Comunidades en las que no existe regulación ninguna y sí existiendo mayoritariamente en el resto -aunque de forma diferente entre éstas en lo que refiere a la identificación de profesiones, actividades y requisitos de acceso-, tendremos como consecuencia un mercado nacional fragmentado y, por tanto, impeditivo de un marco regulador general e igualitario del ejercicio profesional en el deporte (Correa, 2019; García, 2020). A la vez que esta inseguridad jurídica, que determina la falta de referencias únicas en este mercado, embaraza seriamente la uniforme vertebración y articulación de la negociación colectiva nacional en el sector, al diferir las formaciones establecidas por la misma para ejercer las funciones de sus categorías profesionales con las que determinan las distintas 
normativas autonómicas.

Todo lo cual, como insistentemente se ha señalado, sólo puede solventarse con la intervención del Estado sobre la base de la competencia exclusiva de que le dota el artículo 149.1.1 de la Constitución y que le permite el establecimiento de un «mínimo común denominador» (Muñoz, Parejo \& Ruiloba, 1983, p.145) mediante la determinación de un haz de las condiciones básicas de la regulación profesional que tendrán por finalidad, no el diseño acabado y completo de su régimen jurídico, pero sí garantizar la igualdad de todos los operadores en el ejercicio de sus derechos y, por tanto, armonizando su ejercicio profesional en todo el territorio nacional.

Mientras tanto esto no suceda, la regulación de las profesiones del deporte seguirá adoleciendo de una insoportable levedad.

\section{Referencias}

Boletín Oficial de las Cortes Generales (2017), 18 de octubre de 2017. Proposición no de Ley relativa a la regulación y ordenación de las profesiones del Deporte, $n^{\circ} 231$. Recuperado de: http://www.congreso.es/public_oficiales/L12/CONG/DS/CO/ DSCD-12-CO-329.PDF

Bujanda, J. (2017). El reto de la cualificación y profesionalización de los recursos humanos del deporte del País Vasco. Educació Social. Revista d'Intervenció Socioeducativa, 65, 75-93. Recuperado de: https://www.raco.cat/index.php/EducacioSocial/article/view/320557.

Canales-Lacruz, I., \& Rey-Cao, A. (2018). The Socialization Function of Sport in the Election Manifestos of Spanish Political Parties: General Election 2011. Retos. Nuevas Tendencias en Educación Física, Deportes y Recreación, 33, 233-237. Recuperado de: https://recyt.fecyt.es/index.php/retos/article/view/ 57711

Casas, $M^{a}$. E. (2003). La trascendencia constitucional de los principios de ordenación de las fuentes jurídico-laborales. Derecho Privado y Constitución, 17, 89-145.

Cimmino, M. (2018). Servicios de ocio y derecho humano al bienestar. Eunomía, 14, 51-70. DOI:https://doi.org/10.20318/eunomia.2018.4155

Comisión Europea (2011). Desarrollo de la dimensión europea en el deporte, Comunicación al Parlamento Europeo, al Consejo, al Comité Económico y Social Europeo y al Comité de las Regiones, Bruselas. Recuperado de: https://eur-lex.europa.eu/ LexUriServ/LexUriServ.do?uri=COM:2011:0012:FIN:ES:PDF

Consejo COLEF (2017). Las actividades profesionales con exigencia de titulación universitaria en Ciencias de la Actividad Física y del Deporte. Recuperado de: https://drive.google.com/file/d/ 1ebKKouxJQNps5-8IbPf5CYYctGOYFDCe/view

Consejo COLEF (2018). Cinco procesos simultáneos están acaeciendo en estos momentos sin que parezca preocupar a demasiadas partes interesadas de nuestro sector. Recuperado: de https://www.consejo-colef.es/single-post/vgambau-procesoregulacion

Consejo COLEF (2019a). Comunicado sobre las competencias de los/as educadores físico deportivos/as en el ámbito de la salud según el informe del Ministerio de Sanidad, Consumo y Bienestar Social. Recuperado de: https://www.consejo-colef.es/post/ comunicado-competencias-sanidad

Consejo COLEF (2019b). El Consejo COLEF ofrece una propuesta de adaptación del convenio colectivo a las leyes autonómicas de regulación del ejercicio profesional. Recuperado de: https:// www.plataformacolef.es/es/0/1/40/EL-CONSEJO-COLEF-
OFRECE-UNA-PROPUESTA-DE-ADAPTACION-DELCONVENIO-COLECTIVO-A-LAS-LEYES-AUTONOMIC A S - D E - R E G U L A C I O N - D E L - E J E R C I C I O PROFESIONAL.html

Consejo Superior de Deportes (2020). Anuario de Estadísticas Deportivas 2019, Madrid: Ministerio de Cultura y Deporte. Recuperado de: http://www.culturaydeporte.gob.es/dam/ jcr:47414879-4f95-4cae-80c4-e289b3fbced9/anuario-de-estadisticas-deportivas-2020.pdf

Correa, M. (2019). La regulación jurídica del ejercicio de las profesiones del deporte y sus desafíos. Especial referencia a la Ley 5/2016, del Deporte en Andalucía Revista Aranzadi de derecho de deporte y entretenimiento, 64.

Cruz, J. \& Gómez, R. (2014). Artículo 22. Sistema de clasificación profesional. En VVAA., Comentarios al Estatuto de los Trabajadores, Valladolid: Thomson Reuters-Lex Nova.

Espartero, J. \& Palomar, A. (2011). Titulaciones y regulación del ejercicio profesional en el deporte: bases y perspectivas. Madrid: Dykinson.

Espartero, J. (2016). Por la necesaria intervención estatal en la regulación de las profesiones del deporte. Cultura, Ciencia y Deporte, 11(31), 17-26. http://dx.doi.org/10.12800/ ccd.v11i31.639

Espartero, J. (2020). Comentario general a la reciente legislación autonómica sobre profesiones deportivas (755-778). En E. Ortega y M. García (Dirs.), Derecho deportivo 2020. Valencia: Tirant lo Blanch.

Estrada-Marcén, N., Sanz Gonzalo, G., Casterad Seral, J., Simón Grima, J., \& Roso Moliner, A. (2019). Perfil profesional de los trabajadores del sector del fitness en la ciudad de Zaragoza. Retos. Nuevas Tendencias en Educación Física, Deportes y Recreación, 35, 185-190. Recuperado de: https://recyt.fecyt.es/ index.php/retos/article/view/63892

Fernández, D. (2015). Las competencias del Estado como garantía de la unidad de mercado en la jurisprudencia constitucional. Ars Iuris Salmanticensis, 3, 43-76. Recuperado de: https:// revistas.usal.es/index.php/ais/article/view/13886/14325

Fernández, G. (1996). La distribución de competencias entre el Estado y las Comunidades Autónomas. El contenido posible de la Ley básica estatal reguladora de las peculiaridades del régimen jurídico de los Colegios profesionales. En L. Martín (Coord.), Los colegios profesionales a la luz de la Constitución (125-155). Madrid: Unión Profesional-Civitas.

Feu, S., García-Rubio, J., Antúnez, A. \& Ibáñez, S. (2018). Coaching and Coach Education in Spain: A Critical Analysis of Legislative Evolution. International Sport Coaching Journal, 5 (3), 281-292. http://dx.doi.org/10.1123/iscj.2018-0055

Gambau, V. (2019). Acerca de las profesiones y ocupaciones de los titulados universitarios en ciencias de la actividad física y del deporte en España. Revista Española de Educación Física y Deportes, 427, 15-26. Recuperado de: http://www.reefd.es/ index.php/reefd/article/view/840/712

García, J. F., (2020). El acceso al ejercicio de las profesiones deportivas. Revista Aranzadi de Derecho de Deporte y Entretenimiento, 67.

Garrigós, F. (2001). La regulación del ejercicio de la profesión como activo en el desarrollo de la economía del deporte. Revista Internacional de Medicina y Ciencias de la Actividad Física y el Deporte, 1 (3), 188-196. Recuperado de: https:// repositorio.uam.es/xmlui/bitstream/handle/10486/3790/ 26046_3.pdf?sequence $=1$ \&isAllowed $=\mathrm{y}$

Gómez, J. (2019). El deporte adaptado en el Derecho. Madrid: Reus.

González, J.M. \& Contreras, O.R. (2003). Evolución de las tendencias profesionales del titulado en educación física y en ciencias de la actividad física y del deporte desde 1975 hasta la 
actualidad. Apunts. Educación Física y Deportes, 73, 19-23. Recuperado de: https://www.revista-apunts.com/apunts/articulos//73/es/073_019-023ES.pdf

Javaloyes, V. (2019). Obstáculos a la regulación de las profesiones en el deporte. Revista Española de Educación Física y Deportes, 425, 93-102. Recuperado de: https://www.reefd.es/ index.php/reefd/article/download/743/631

Jiménez, I. (2001). El ejercicio profesional de las titulaciones del deporte. Barcelona: Bosch.

Landaberea, J. A. (2008). Cuestiones competenciales de la regulación del ejercicio de las profesiones del deporte. A propósito de la Ley catalana 3/2008, de 23 de abril. Revista Jurídica del Deporte, 24, 191-211. Recuperado de: http://www.kirolan.org/ D o c u m e n o s \% 20 o b s e r v a tori o LeyprofesionesdeportecatalunaJuantxoLandaberea.pdf

Lolli, S. (1997). Le professioni dello sport: la situazione italiana. Milano: Franco Angeli.

Madrera E., González, A., \& Esteban, L. (2015). La formación de profesionales de las actividades físico-deportivas en la enseñanza no-universitaria. Retos. Nuevas Tendencias en Educación Física, Deportes y Recreación, 27, 152-158. Recuperado de: https://dialnet.unirioja.es/descarga/articulo/5407734.pdf

Martín, M., Espada, M., Moscoso, D., Jiménez, J.E., Santacruz, J.A., \& Jiménez, V. (2018). La práctica de actividad física y deporte: una demanda sociológicamente construida. Revista de Humanidades, 34, 87-105. Recuperado de: https:// dialnet.unirioja.es/descarga/articulo/6462047.pdf

Martínez-Lemos, R. I., \& Hontoria-Hernández, E. (2019). El efecto tamaño y sector sobre la rentabilidad de las empresas vinculadas al deporte en España. Retos. Nuevas Tendencias en Educación Física, Deportes y Recreación, 37, 160-166. https:// doi.org/10.47197/retos.v37i37.69480

Miñana-Signes, V., \& Monfort-Pañego, M. (2020). Justificación del valor educativo de la Educación Física y el docente. ¿Qué profesional del deporte debe impartir la materia de Educación Física? ¿El Maestro/a y Profesor/a de Educación Física o el Monitor/a deportivo/a? Retos. Nuevas Tendencias en Educación Física, Deportes y Recreación, 38, 852-867. https://doi.org/ 10.47197/retos.v38i38.74722

Muñoz, D. (2018). Comentarios a la Ley de la Actividad Física y el Deporte de Castilla-La Mancha: novedades, reflexiones y análisis comparativo. Cuenca: Universidad de Castilla la Mancha.

Muñoz, S., Parejo, L. \& Ruiloba, E. (1983). La libertad de ejercicio de la profesión y el problema de las atribuciones de los técnicos titulados. Madrid: IEAL.

Palomar, A. (2000). La regulación de las titulaciones deportivas en el ámbito del Real Decreto 1930/1997, de 19 de diciembre. Revista Aranzadi de Derecho de Deporte y Entretenimiento, 3 , 13-59.

Palomar, A. (2019). Viabilidad de una ley estatal ordenadora de la profesión. Revista Española de Educación Física y Deportes, 425, 103-130. Recuperado de: https://www.reefd.es/index.php/ reefd/article/view/744/632

Parlamento Europeo (2018). Directiva 2018/958/UE, de 28 de junio, relativa al test de proporcionalidad antes de adoptar nuevas regulaciones de profesiones. Recuperado de: https:// www.boe.es/doue/2018/173/L00025-00034.pdf

Pascual, A. (2016). La competencia autonómica en materia de profesiones del deporte: la Ley 1/2015, de 23 de marzo, del ejercicio físico y del deporte de La Rioja. Revista General de Derecho Constitucional, 22.

Pastor, J. L. (1997). El espacio profesional de la educación física en España: Génesis y formación (1883-1961). Alcalá de Henares: Universidad de Alcalá.

Sánchez, A. \& Rebollo, S. (2000). Situación del mercado laboral actual en el ámbito de la actividad física y deportiva. Revista
Motricidad, 6, 141-154. Recuperado de: http:// www.eurjhm.com/index.php/eurjhm/article/download/59/117

Sánchez, C. (2012). Actividad física, deporte y profesión: aproximación jurídica. Actividad Física y Deporte: Ciencia y Profesión, 17, 23-31. Recuperado de: http://colefcafecv.com/wpcontent/uploads/2015/04/Revista-n\%C2\%BA17.-Actividadf\%C3\%ADsica-deporte-y-proximaci\%C3\%B3n.Aproximaci\%C3\%B3n-jur\%C3\%ADdica.pdf

Sánchez, L. (2019). El Derecho y las profesiones. Revista Española de Educación Física y Deportes, 425, 65-91. http:// www.reefd.es/index.php/reefd/article/view/742/630

Sans, J., \& Inglés, E. (2020). Análisis comparativo de la legislación en materia de las titulaciones de Técnico Deportivo y Técnico Deportivo Superior en la especialidad de deportes de invierno en España. Retos. Nuevas Tendencias en Educación Física, Deportes y Recreación, 37, 351-361. Recuperado de: https:// recyt.fecyt.es/index.php/retos/article/view/72606

STC 109/2003, de 5 de junio. Recuperado de: http://www.boe.es/ boe/ dias/2003/07/01/pdfs/T00073-00096.pdf

STC 111/1993, de 25 de marzo. Recuperado de: http://www.boe.es/ diario_boe/txt.php?id=BOE-T-1993-10694

STC 122/1989, de 6 de julio. Recuperado de: http://www.boe.es/ diario_boe/txt.php?id=BOE-T-1989-17487

STC 154/2005, de 9 de junio. Recuperado de: de http://www.boe.es/ diario_ boe/txt.php?id=BOE-T-2005-11741

STC 194/1998, de 1 de octubre de 1998. Recuperado de: https:// www.boe.es/diario_boe/txt.php?id=BOE-T-1998-24940

STC 194/1998, de 1 de octubre. Recuperado de http://www.boe.es/ diario_ boe/txt.php?id=BOE-T-1998-24940

STC 201/2013, de 5 de diciembre. Recuperado de: http://boe.es/ diario_boe/txt.php?id=BOE-A-2014-218

STC 210/1990, de 20 de diciembre. Recuperado de: https:// www.boe.es/buscar/doc.php?id=BOE-T-1991-613

STC 212/2005, de 21 de julio de 2005. Recuperado de: https:// www.boe.es/buscar/doc.php?id=BOE-T-2005-14163

STC 31/2010, de 28 de junio de 2010. Recuperado de: http://boe.es/ diario_boe/txt.php?id=BOE-A-2010-11409

STC 330/1994, de 15 de diciembre. Recuperado de: https:// www.boe.es/buscar/doc.php?id=BOE-T-1995-1225

STC 42/1981, de 22 de diciembre. Recuperado de: http:// www.boe.es/ diario_boe/txt.php?id=BOE-T-1982-965

STC 42/1986, de 10 de abril. Recuperado de: http://www.boe.es/ diario_boe/txt.php?id=BOE-T-1986-10619

STC 82/1986, de 26 de junio. Recuperado de: http://www.boe.es/ diario_ boe/txt.php?id=BOE-T-1986-17827

STC 83/1984, de 24 de julio. Recuperado de: http://www.boe.es/ boe/ dias/1984/08/24/pdfs/T00011-00015.pdf

STS 3127/2018, de 17 de julio. Recuperado de: http:// www.poderjudicial.es/search/openCDocument/ e5e0cf323aea82eb599e4e9439214f915f47ce064112b670

Terol, R. (2019). Cualificaciones profesionales y Derecho de la Unión Europea. Amministrativ@mente Rivista Scientifica di Diritto Amministrativo, 3. Recuperado de: http:// www.amministrativamente.com/index.php/formez/article/view/ 13128

Tornos, J. (2014). La ley 20/2013, de 9 de diciembre, de garantía de la unidad de mercado. En particular, el principio de eficacia. Revista d'Estudis Autonòmics i Federals, 19, 144-177.

Zaragozá-Sevilla, J., \& Martínez-Baena, A. (2018). Conectando teoría y práctica profesional en el Grado en Ciencias de la Actividad Física y el Deporte. Revista Española de Educación Física y Deportes, 420, 39-57. Recuperado de: http:// www.reefd.es/index.php/reefd/article/view/645 